\title{
Modeling Sentence Comprehension Deficits in Aphasia: A Computational Evaluation of the Direct-Access Model of Retrieval
}

\author{
Paula Lissón, Dorothea Pregla, Dario Paape, \\ Frank Burchert, Nicole Stadie, Shravan Vasishth \\ University of Potsdam \\ \{lissonhe, pregla, paape, nstadie, burchert, vasishth\}@uni-potsdam.de
}

\begin{abstract}
Several researchers have argued that sentence comprehension is mediated via a contentaddressable retrieval mechanism that allows fast and direct access to memory items. Initially failed retrievals can result in backtracking, which leads to correct retrieval. We present an augmented version of the directaccess model that allows backtracking to fail. Based on self-paced listening data from individuals with aphasia, we compare the augmented model to the base model without backtracking failures. The augmented model shows quantitatively similar performance to the base model, but only the augmented model can account for slow incorrect responses. We argue that the modified direct-access model is theoretically better suited to fit data from impaired populations.
\end{abstract}

\section{Introduction}

Comprehending a sentence involves building linguistic dependencies between words. In the sentence processing literature, several researchers have argued that linguistic dependency resolution is carried out via a cue-based retrieval mechanism (Van Dyke and McElree, 2006; Lewis and Vasishth, 2005). Cue-based retrieval theory assumes that word representations are retrieved from working memory via their syntactic and semantic features. Consider the following sentences:

(1) a. The boy who tickled the girl greeted the teacher.

b. The boy who the girl tickled greeted the teacher.

In (1a), the noun boy would be encoded in memory with features such as [+animate, +subj]. When the reader reaches the verb tickled, a retrieval is triggered with retrieval cues that match the features of boy. At this point in time, boy is the only element that matches the retrieval cues of the verb. By contrast, in (1b), another noun intervenes between tickled and boy that partially matches the cues set at the retrieval: girl [+animate, -subj]. The partial feature overlap causes similarity-based interference between the two items, making the dependency more difficult to resolve in (1b) compared to (1a).

Interference effects have been attested in multiple studies, see for example Jäger et al. (2020); Gordon et al. (2006); Jäger et al. (2017); Van Dyke (2007). One model of cue-based retrieval that predicts these interference effects is the directaccess model developed by McElree and colleagues (McElree, 2000; McElree et al., 2003; Martin and McElree, 2008). The direct-access model (DA) assumes that retrieval cues allow parallel access to candidate items in memory, as opposed to a serial search mechanism. Due to the parallelism assumption, the speed of retrieval is predicted to be constant across items (aside from individual differences and stochastic noise in the retrieval process).

Factors such as increased distance between the target and the retrieval point and the presence of distractor items can lower the probability of retrieving the correct dependent (also known as availability). Low availability of the target dependent can lead to failures in parsing or to misretrievals of competitor items. When such errors occur, a backtracking process can be initiated, which by assumption leads to the correct retrieval of the target (McElree, 1993). The backtracking process requires additional time that is independent of the retrieval time. According to the direct-access model, (1a) should have shorter processing times than (1b) on average, because in (1b) some trials require costly backtracking due to lower availability of the target item boy.

The direct-access model can be adapted to explain impaired sentence comprehension in individuals with aphasia (IWA; Lissón et al., 2021). However, there is one crucial aspect of the directaccess model that is at odds with the aphasia literature, specifically with the finding that IWA have 
longer processing times for incorrect than for correct responses (e.g., Hanne et al., 2015; Pregla et al., 2021). The direct-access model assumes that some percentage of correct interpretations are only obtained after costly backtracking, and thus predicts that the average processing time for incorrect responses should be faster than for correct responses. To address this issue, we implement a modified version of the direct-access model that is specifically relevant for sentence processing in IWA. In this model, backtracking can lead to correct retrieval of the target, as in the base model, but can also result in misretrieval and parsing failure.

\subsection{Sentence Comprehension in Aphasia}

Aphasia is an acquired neurological disorder that causes language production and comprehension impairments. In the aphasia literature, there are several theories that aim to explain the source of these impairments in language comprehension. One possibility is that IWA carry out syntactic operations at a slower-than-normal pace, which could cause failures in parsing. This is the slow syntax theory (Burkhardt et al., 2008). By contrast, Ferrill et al. (2012) claim that the underlying cause of slowed sentence processing in IWA is delayed lexical access, which cannot keep up with structure building. Another theory, resource reduction, assumes that IWA experience a reduction in the resources used for parsing (Caplan, 2012), such as working memory. Finally, Caplan et al. (2013) claim that IWA suffer from intermittent deficiencies in their parsing system that lead to parsing failures. Previous computational modeling work has shown that these theories may be complementary (Patil et al., 2016; Lissón et al., 2021), and that IWA may experience a combination of all of these deficits (Mätzig et al., 2018).

Assuming that a direct-access mechanism of retrieval subserves sentence comprehension, this mechanism could interact with one or more of the proposed processing deficits in IWA. One way to assess whether these deficits are plausible under a direct-access model is the computational modeling of experimental data. Lissón et al. (2021) tested the direct-access model against self-paced listening data from individuals with aphasia, finding the model to be in line with multiple theories of processing deficits in aphasia. Despite this encouraging result, the model could not fit slow incorrect responses, due to its assumptions about backtrack- ing and its consequences.

In what follows, we present our implementation of the original direct-access model and the modified version with backtracking failures. We fit the two models to data from individuals with aphasia and compare their quantitative performances. In order to assess the role of the different proposed deficits of IWA in sentence comprehension, we also map the models' parameters onto theories of processing deficits in aphasia.

\section{Data}

The data that we model come from a self-paced listening task in German (Pregla et al., 2021). 50 control participants and 21 IWA completed the experiment. Sentences were presented auditorily, word by word. Participants paced the presentation themselves, choosing to hear the next word by pressing a computer key. The time between key presses (here called listening time) was recorded. At the end of the sentence, two images (target and foil) were presented, and participants had to select which image matched the meaning of the sentence they had just heard. Accuracies for the picture-selection task were also recorded. To assess test-retest reliability, each subject completed the task twice, with a break of two months in between. Our modeling is based on the pooled data of both sessions.

\subsection{Items}

We investigate interference effects in a linguistic construction that is understudied in IWA: Control constructions. In control constructions, the subject of an infinitival clause is not overly specified, but understood to be coreferential with one of the overt noun phrases in the matrix clause of the same sentence (e.g, Brian promises Martha to take out the trash $\rightarrow$ Brian takes out the trash). In linguistic theory, it is assumed that a a phonologically empty element (PRO) occupies the subject position of take out (Chomsky, 1981). PRO is co-indexed with a noun phrase in the matrix clause that acts as its antecedent. The verb in the matrix clause specifies, according to its semantic and syntactic properties, which noun phrase in the matrix clause triggers the interpretation of PRO in the subclause.

In sentence (2a) below, the verb verspricht (promises) is lexically specified as a subjectcontrol verb, and the subject noun phrase of the main clause, Peter, is chosen as the antecedent of PRO. By contrast, in (2b), the object-control 
verb erlaubt (allows) specifies that the object noun phrase of the main clause, Lisa, is the antecedent of PRO.

\section{(2) a. Subject control}

Peter $_{i}$ verspricht nun $\mathrm{Lisa}_{\mathrm{j}}, \mathrm{PRO}_{\mathrm{i}}$ das kleine Lamm $\mathrm{zu}$ streicheln und $\mathrm{zu}$ kraulen.

'Peter now promises Lisa to pet and to ruffle the little lamb'

b. Object control

Peter $r_{i}$ erlaubt nun $\mathrm{Lisa}_{\mathrm{j}}, \mathrm{PRO}_{\mathrm{j}}$ das kleine Lamm $\mathrm{zu}$ streicheln und $\mathrm{zu}$ kraulen.

'Peter now allows Lisa to pet and to ruffle the little lamb'

Cue-based retrieval theory assumes that control clauses require completion of the PRO dependency through memory access to the correct noun phrase. The direct-access model would predict ( $2 b)$ to be easier to process than (2a), because the target (Lisa) is linearly closer to the retrieval site at PRO, and thus more available. Therefore, at PRO, the probability of retrieval of the target should be higher in (2b) relative to (2a). In line with this prediction, unimpaired subjects show a processing advantage for object control over subject control (Kwon and Sturt, 2016). Similarly, IWA exhibit more difficulties understanding subject control conditions in acting-out tasks (Caplan and Hildebrandt, 1988; Caplan et al., 1996). However, the object control advantage in IWA has not been previously tested using online methods.

Our experimental items were 20 sentences (10 per condition) similar to (2a) and (2b). The corresponding pictures for the picture-selection task are shown in Figure (1). The top picture is the target picture for (2a), whereas the bottom picture is the target for (2b). We assume that trials where the foil picture has been selected (i.e., the picture that shows the distractor noun as the agent of the action) correspond to a misretrieval.

\subsection{Dependent Variables}

The dependent variables used for modeling were the listening times (henceforth, LT) at the retrieval site (PRO) and the accuracy of the picture-selection task. Given that PRO is phonologically empty, we assumed that the retrieval process takes place at some point between the second and the third noun phrase (Lisa and das kleine Lamm in (2a)). We
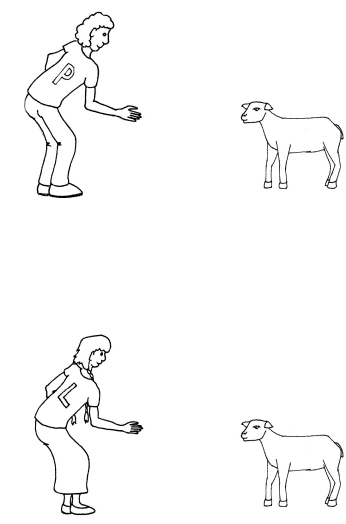

Figure 1: Example pictures used in the pictureselection task.

therefore summed the listening times of these regions within each trial. In order to evaluate the slowed lexical access hypothesis (Ferrill et al., 2012), we also used data from an auditory lexical decision task that participants performed in addition to the experiment. This task was based on LEMO 2.0 (Stadie et al., 2013). Participants had to decide whether an auditorily presented item was a word or a neologism, and the response times were recorded. For each participant, we computed the mean response times for correct responses. These were then centered and scaled within groups and used as continuous predictors in the models. We will refer to the scaled lexical decision task reaction times as the $L D T$ predictor.

\section{Direct-Access Model}

Our implementation of the direct-access model follows Nicenboim and Vasishth (2018). The model assumes that listening times for correct responses come from a mixture distribution, given that there are trials with backtracking, where an additional processing $\cos t \delta$ is added, and trials without backtracking, where no such cost is added. By contrast, incorrect responses never involve backtracking, and the average listening time should be the same as for correct responses without backtracking. A graphical representation of the model is displayed in Figure (2). The three possible cases are as follows:

(a) Retrieval of the target succeeds at first attempt, with probability $\theta$ :

$$
L T \sim \operatorname{lognormal}(\mu, \sigma)
$$




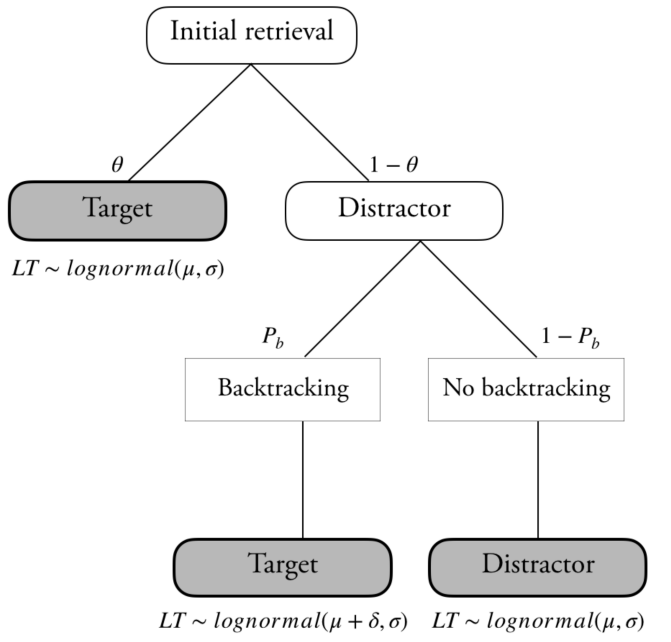

Figure 2: Graphical representation of the direct-access model.

(b) Retrieval fails at first attempt, backtracking is initiated, with probability

$(1-\theta) \cdot P_{b}: L T \sim \operatorname{lognormal}(\mu+\delta, \sigma)$

(c) Retrieval fails, no backtracking, and a misretrieval occurs, with probability

$(1-\theta) \cdot\left(1-P_{b}\right): L T \sim \operatorname{lognormal}(\mu, \sigma)$

The model includes both fixed and random effects in order to account for sentence complexity, group differences, and individual variability. The hierarchical structure is shown in Equation (1). All parameters have an adjustment by group (IWA versus control), because we expect IWA to have different parameter estimates from control participants. Since DA assumes that retrieval times are not affected by sentence complexity, the average listening times $(\mu)$ do not have an adjustment for condition. By contrast, the probability of retrieval of the target, $\theta$, includes a condition adjustment. This parameter can be thought of as indexing memory availability. The probability of backtracking $P_{b}$, the cost of backtracking $\delta$, and $\sigma$ do not depend on sentence complexity, but may vary between IWA and controls. The hierarchical structure is embedded within the parameters when possible (we report the maximal hierarchical structure that could be fit). In Equation (1), the terms $u$ and $w$ are the by-participant and by-item adjustments to the fixed effects, respectively. These are assumed to come from two multivariate normal distributions. All parameters had regularizing priors, listed in Appendix B.

$$
\begin{array}{r}
\mu=\mu_{0}+u_{\mu 0}+w_{\mu 0}+\beta_{1} \cdot \text { group } \\
\theta=\alpha+u_{\alpha}+w_{\alpha}+\beta_{2} \cdot \text { LDT }+ \\
\beta_{3} \cdot L D T \cdot \text { group }+ \\
\left(\beta_{4}+w_{\beta_{4}}\right) \cdot \text { group } \\
\left(\beta_{5}+u_{\beta_{5}}\right) \cdot \text { condition }+ \\
\beta_{6} \cdot \text { group } \cdot \text { condition } \\
P_{b}=\gamma+u_{\gamma}+\beta_{7} \cdot \text { group } \\
\delta=\delta_{0}+\beta_{8} \cdot \text { group } \\
\sigma=\sigma_{0}+\beta_{9} \cdot \text { group }
\end{array}
$$

The model was implemented in the probabilistic programming language Stan (Stan Development Team, 2020), and fit via the rstan package (Carpenter et al., 2017) in R (R Core Team, 2020). The model was fit with 3 chains and 8,000 iterations, half of which were used as warm-up.

\subsection{Predictions}

Based on the theories of processing deficits in aphasia discussed in Section (1.1), and on the findings in Lissón et al. (2021), we make the following predictions:

1. IWA's $\mu$ and $\delta$ values should be higher than controls'. This would be in line with slow syntax, assuming that both the initial retrieval and the backtracked retrieval are accompanied by appropriate structure-building processes.

2. The probability of initial retrieval of the target $\theta$ should be lower for IWA relative to controls, across conditions.

3. Object control conditions should have a larger $\theta$, relative to subject control. In addition, IWA should have a bigger interference effect, i.e., the difference in $\theta$ between the two conditions should be larger in IWA than in controls. This pattern would be expected under the resource reduction theory, which states that IWA should have greater difficulties in more complex sentences.

4. Slower lexical decision (LDT) should be associated with a decrease in $\theta$ across groups. Strong support for delayed lexical access would come from an interaction between LDT and group, such that an increase in LDT predicts a greater decrease in $\theta$ for IWA than for controls: Slow lexical access could cause 
parsing problems for controls, but if delayed lexical access is the main cause of difficulty in IWAs, parsing failures should occur more often in this group for individuals whose lexical access is particularly slow.

5. The probability of backtracking should be lower for IWA, which would be in line with resource reduction.

6. Finally, the dispersion parameter $\sigma$ of the listening-time distribution should be larger for IWA, which would indicate that IWA have more noise in their parsing system. This would be in line with intermittent deficiencies, since more noise could be due to more breakdowns in parsing.

These predictions build on the previous work by Lissón et al. (2021), but other options for the mapping between parameters and theories of comprehension deficits in aphasia are possible, see Mätzig et al. (2018); Patil et al. (2016).

\subsection{Results}

We begin by assessing the posterior distribution of the probability of retrieval of the target, $\theta$, shown in Figure (3).

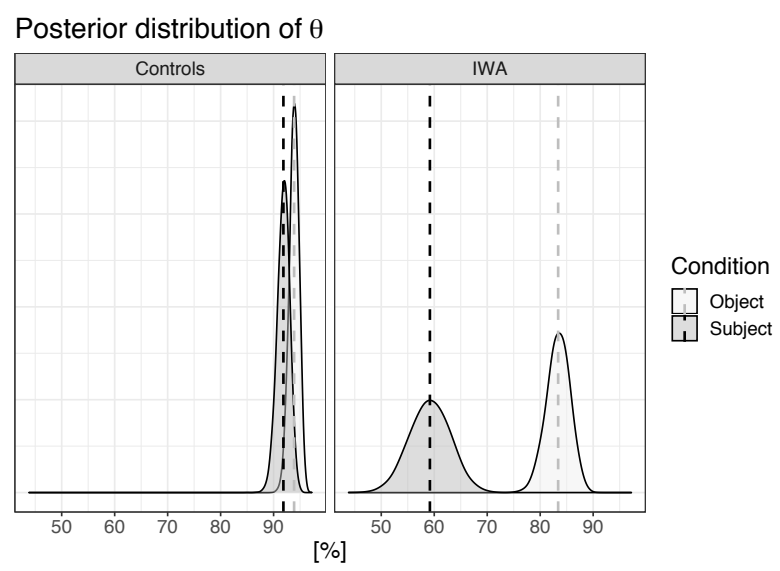

Figure 3: Posterior distribution of $\theta$ across conditions and groups.

Controls are estimated to retrieve the target at the first retrieval attempt in both conditions in more than $90 \%$ of trials. The mean of the subject-control condition is slightly lower than the mean for the object-control condition. By contrast, IWA display a greater effect of interference: In object-control sentences, where the antecedent is close to PRO, IWA are estimated to correctly retrieve the target at the first attempt $85 \%$ of the time, compared to
$60 \%$ for subject-control. An increase in LDT leads to a decrease in $\theta$ of $-6 \% \mathrm{CrI}$ : [-11\%, $-2 \%]$, but there was no interaction with group $\times$ LDT $(-2 \%$ CrI: $[-6 \%, 2 \%])$. The credible intervals for the remaining parameters are shown in Table (1).

\begin{tabular}{lll}
\hline Par. & Control participants & IWA \\
\hline$\mu$ & {$[1668 \mathrm{~ms}, 1901 \mathrm{~ms}]$} & {$[2508 \mathrm{~ms}, 3073 \mathrm{~ms}]$} \\
$\delta$ & {$[1084 \mathrm{~ms}, 1385 \mathrm{~ms}]$} & {$[2897 \mathrm{~ms}, 6836 \mathrm{~ms}]$} \\
$P_{b}$ & {$[63 \%, 78 \%]$} & {$[3 \%, 10 \%]$} \\
$\sigma$ & {$[0.15,0.16]$} & {$[0.27,0.3]$} \\
\hline
\end{tabular}

Table 1: Parameter credible intervals, DA model.

As expected under the slow syntax theory, IWA's mean listening times $(\mu)$ and the time needed for backtracking $(\delta)$ are higher than controls'. Similarly, $\sigma$ is also higher for IWA, as predicted by intermittent deficiencies. Finally, the probability of backtracking is much lower for IWA than for controls. Assuming that backtracking uses general parsing resources, this estimate is in line with resource reduction.

\subsection{Posterior Predictive Checks}

One way to assess the behavior of the model is to check the posterior distribution of data generated by the model against the empirical data. If the mean of the empirical data falls within the range of predicted values of the model, the model could have generated the empirical data. By contrast, if the empirical data are outside of the range of the generated values, this indicates a suboptimal fit. Figure (4) shows the posterior predictive distributions of the direct-access model across groups and conditions. Overall, correct responses are modeled reasonably well, except in the object-control condition for IWA. The model also underestimates the listening times for incorrect responses, except for IWA in the subject-control condition. In all other design cells, incorrect responses are slower than correct responses, contrary to the model's assumption that slow backtracking responses are always correct.

\section{Modified Direct-Access Model}

Based on the original DA model's suboptimal fit, we propose a modified version (MDA). In this version, the distribution of listening times for both correct and incorrect responses is a mixture of directly accessed and backtracked retrievals. The MDA model assumes that backtracking can fail. 


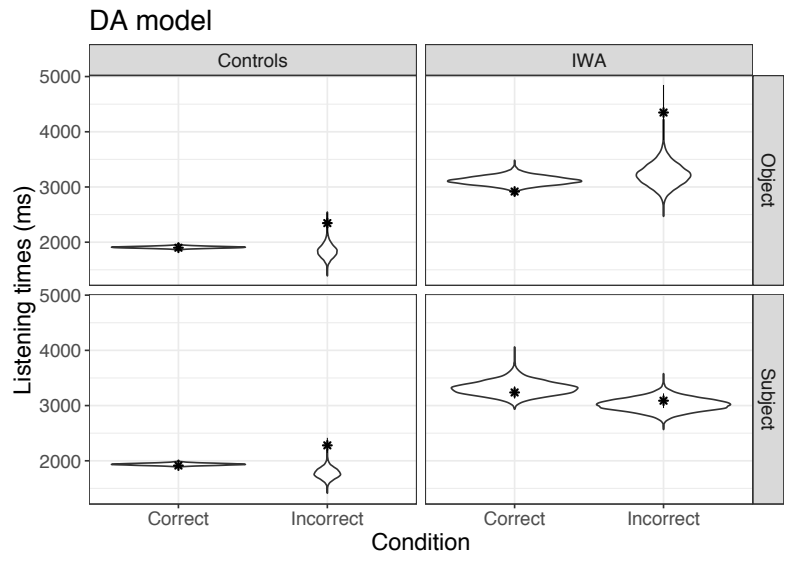

Figure 4: Posterior predictive checks of the directaccess model split by accuracy, group, and condition. The violin plots indicate the distribution of listening times generated by the model. The black stars stand for the mean of the empirical data.

In terms of implementation, the main difference between the models is a newly-introduced parameter $\theta_{b}$, which is the probability of correct retrieval after backtracking. Figure (5) displays a graphical representation of this new model: After backtracking, the target is retrieved with probability $\theta_{b}$, and a misretrieval occurs with probability $1-\theta_{b}$. The hierarchical structure is the same as in the DA original model, except for $\theta_{b}$, whose adjustments are shown in Equation (2).

$$
\theta_{b}=\alpha_{b}+u_{\alpha_{b}}+\beta \cdot \text { group }
$$

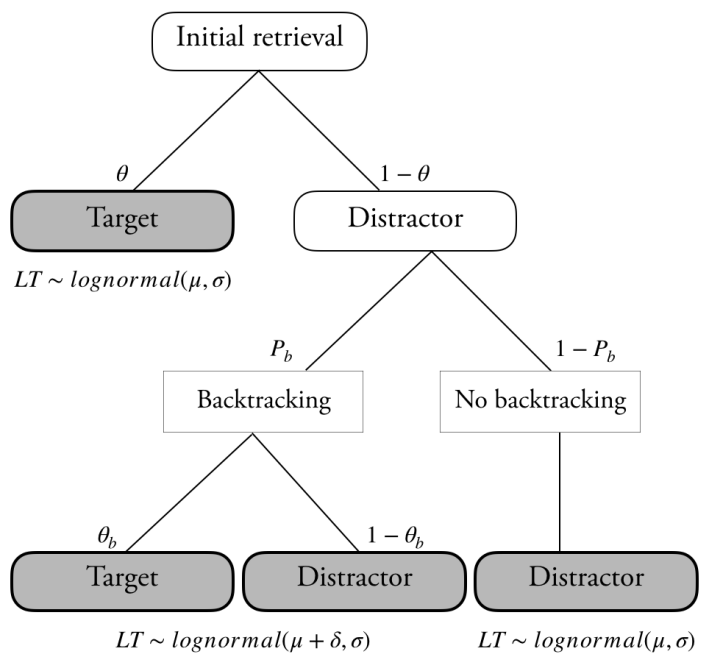

Figure 5: Graphical representation of the modified direct-access model.

The model was run with 10,000 iterations, half of which were used as warm-up.

\subsection{Predictions}

All predictions are carried over from the base DA model. In addition, the probability of retrieval of the target after backtracking $\theta_{b}$ should be lower for IWA than for controls. This would indicate that IWA are more likely to experience parsing failure or misretrieval even after backtracking.

\subsection{Results}

We begin by assessing the probability of first correct retrieval, $\theta$. The posterior distribution across groups and conditions is shown in Figure (6). The estimates are quite similar to the ones in the original DA model: Controls have a very high probability of initial correct retrieval across conditions, and IWA display a greater interference effect.

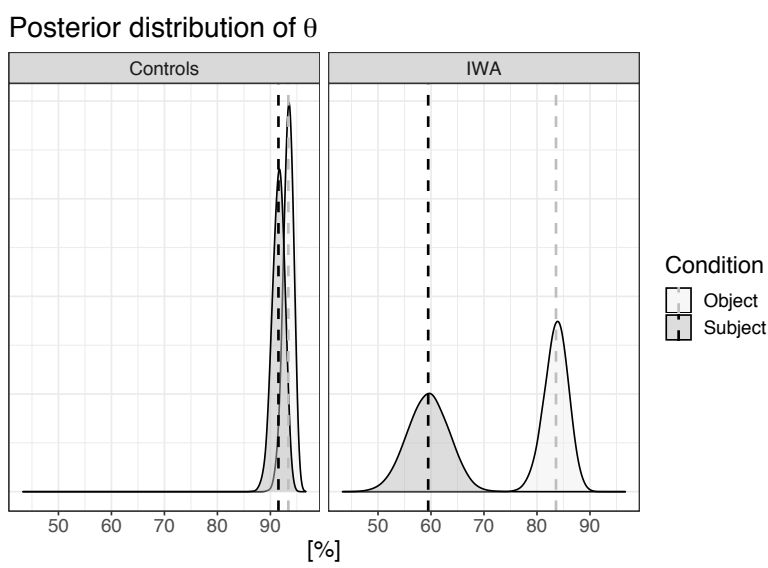

Figure 6: Posterior distribution of $\theta$ across conditions and groups.

As in the base model, IWA have a low probability of backtracking in this model (7\% CrI: [4\%, $12 \%])$ relative to controls (80\%, CrI: [72\%, $86 \%])$. The probability of correct retrieval after backtracking, $\theta_{b}$, determines the amount of slow incorrect responses. The posterior distribution of $\theta_{b}$ is shown in Figure (7). After backtracking, controls are estimated to retrieve the target $90 \%$ of the time, compared to around $70 \%$ for IWA.

The rest of estimates are also similar to the ones in the original DA model: IWA's $\mu$ is higher than controls' (2751 ms, CrI: [2477, 3046] versus $1770 \mathrm{~ms}$, CrI: [1654 ms, $1890 \mathrm{~ms}])$. The cost of backtracking, $\delta$, is very high for IWA (6394 ms CrI: [4235, 9468]) relative to controls $(1238 \mathrm{~ms}$, CrI: [1103 ms, $1387 \mathrm{~ms}])$. Finally, $\sigma$ is also higher for IWA (0.27 CrI: $[0.25,0.28])$ than for controls $(0.15$ CrI: [0.14, 0.15]). 
Posterior distribution of $\theta_{b}$

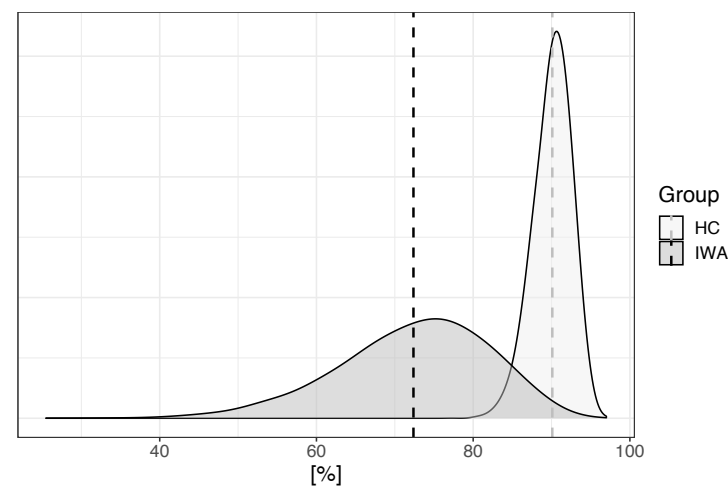

Figure 7: Posterior distribution of $\theta_{b}$ across conditions and groups.

\subsection{Posterior Predictive Checks}

The posterior predictive checks for the modified direct-access model are shown in Figure (8).

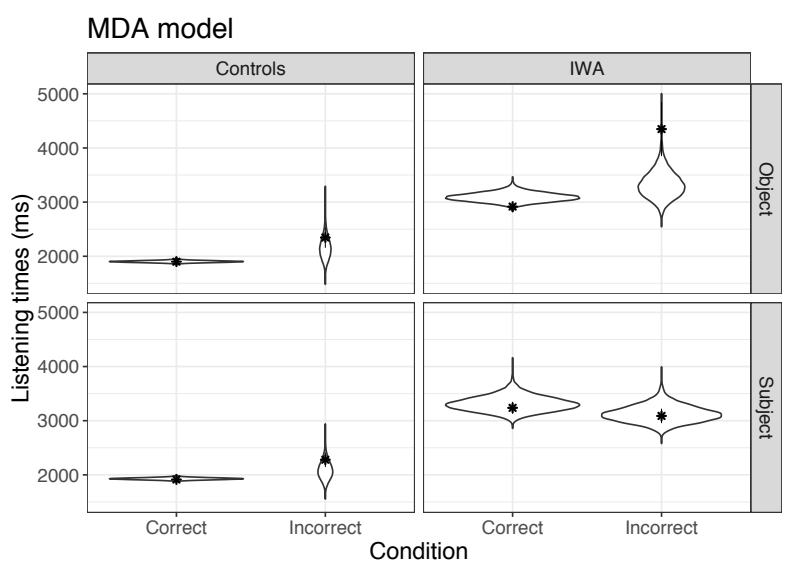

Figure 8: Posterior predictive checks of the modified direct-access model split by accuracy, group, and condition. The violin plots indicate the distribution of listening times generated by the model. The black stars stand for the mean of the empirical data.

Like the base model, the MDA mostly correctly estimates listening times for correct responses across the board. The fits for incorrect responses seem to have improved, except for object-control in IWA, where the predicted listening times are still faster than the observed listening times.

\section{Model Comparison}

In order to quantitatively compare the performance of the models, we computed Bayes factors. We chose Bayes factors over other alternatives (e.g. cross-validation), because the two models seem to predict similar distributions, and Bayes factors are especially suited for nested models, or models that make very similar predictions. The hypothesis being tested is whether there is a non-zero parameter $\theta_{b}$ that indexes the probability of successful backtracking, assumed by the MDA model, or whether backtracking is always successful, as assumed by the base DA model.

In order to perform the comparison, the models were run for 40,000 iterations, of which 3,000 were used for warm-up. Bayes factors were computed using the bridgesampling package (Gronau et al., 2020 ) in R. The Bayes factor of DA over MDA was estimated to be 2. This result is inconclusive, and indicates that the models provide similar quantitative fit to the data.

\section{Discussion and Conclusion}

In the present paper, we implemented and tested two versions of the direct-access model of cuebased retrieval and evaluated their predictive performance on data from individuals with aphasia and control participants. Specifically, we modeled interference in an under-studied linguistic construction, namely control structures.

Both the base model and the modified model are in line with a combination of processing deficits in IWA: slow syntax, resource reduction, and intermittent deficiencies. Neither of the two models showed support for delayed lexical access as a source of retrieval difficulty specifically for IWA. Although a delay in LDT was connected to a decrease in the probability of correct retrieval, the effect of LDT was similar for IWA and control participants. In general, our results are consistent with other studies showing that a combination of processing deficits may be the source of impairments in sentence comprehension in IWA (Caplan et al., 2015; Mätzig et al., 2018; Lissón et al., 2021).

Unlike the base direct-access model, our modified DA model (MDA) assumes that backtracking can fail, resulting in slow, incorrect retrievals. However, this added assumption does not result in a decisive advantage in fit for the MDA model, as shown by the posterior predictive checks and the Bayes factor analysis. This result is unexpected, and leads us to think that the MDA model may be overparametrized. In MDA, all of the main parameters include a group adjustment. As a consequence, for instance, the mean listening times, $\mu$, are estimated to be higher for IWA than for controls. The cost of backtracking, which is only added to $\mu$ if backtracking is performed, accounts for slower re- 
sponses. However, because IWA's $\mu$ is estimated to be higher than controls' $\mu$, the model may not need to rely on backtracking in order to account for slow responses in IWA. This could be the reason why the probability of backtracking for IWA is very low $(7 \%)$ relative to controls (80\%). In addition, IWA's $\theta_{b}$ has to be estimated from the $7 \%$ of trials that include backtracking. Given the size of the IWA group (21 participants), and the small amount of trials that include backtracking, perhaps the model cannot correctly estimate the $\theta_{b}$ parameter. This could be investigated in several ways. One possibility would be to remove the group adjustments from $\mu, P_{b}, \delta$, and $\theta_{b}$ one at the time, and see which of these models shows a better quantitative fit for the data (see Lissón et al., 2021). Another possibility would be to evaluate how these parameters interact with and without group adjustments (e.g., do $P_{b}$ and/or $\delta$ for IWA increase if there is no group adjustment in $\mu$ ?). We will address these questions in future work.

The present paper contributes to the aphasia literature by proposing a modification of the directaccess model that can account for incorrect slow responses. Despite our inconclusive results, we believe that the modified direct-access model offers a more appropriate set of assumptions for individuals with aphasia than the direct-access model. The modified-direct access model can account for slow incorrect responses, which are frequently found in studies on sentence processing in IWA (e.g., Hanne et al., 2015; Lissón et al., 2021; Pregla et al., 2021). It remains to be seen, by testing the new modified direct-access model against more data from individuals with aphasia, whether there is a difference in predictive performance between the two models.

\section{References}

Petra Burkhardt, Sergey Avrutin, Maria M. Piñango, and Esther Ruigendijk. 2008. Slower-than-normal syntactic processing in agrammatic broca's aphasia: Evidence from Dutch. Journal of Neurolinguistics, 21(2):120-137.

David Caplan. 2012. Resource reduction accounts of syntactically based comprehension disorders. In C. K. Thompson and R. Bastiannse, editors, Perspectives on Agrammatism, pages 34-48. Psychology Press, London, New York.

David Caplan and Nancy Hildebrandt. 1988. Disorders of syntactic comprehension. MIT Press, Cambridge.

David Caplan, Nancy Hildebrandt, and Nikos Makris.
1996. Location of lesions in stroke patients with deficits in syntactic processing in sentence comprehension. Brain, 119(3):933-949.

David Caplan, Jennifer Michaud, and Rebecca Hufford. 2013. Dissociations and associations of performance in syntactic comprehension in aphasia and their implications for the nature of aphasic deficits. Brain and Language, 127(1):21-33.

David Caplan, Jennifer Michaud, and Rebecca Hufford. 2015. Mechanisms underlying syntactic comprehension deficits in vascular aphasia: new evidence from self-paced listening. Cognitive Neuropsychology, 32(5):283-313.

Bob Carpenter, Andrew Gelman, Matthew D. Hoffman, Daniel Lee, Ben Goodrich, Michael Betancourt, Marcus Brubaker, Jiqiang Guo, Peter Li, and Allen Riddell. 2017. Stan: A probabilistic programming language. Journal of Statistical Software, 76(1):1-32.

Noam Chomsky. 1981. Lectures on government and binding. Foris, Dordrecht.

Michelle Ferrill, Tracy Love, Matthew Walenski, and Lewis P. Shapiro. 2012. The time-course of lexical activation during sentence comprehension in people with aphasia. American Journal of SpeechLanguage Pathology, 21(2):S179.

Peter C Gordon, Randall Hendrick, Marcus Johnson, and Yoonhyoung Lee. 2006. Similarity-based interference during language comprehension: Evidence from eye tracking during reading. Journal of experimental Psychology: Learning, Memory, and Cognition, 32(6):1304-1321.

Quentin F. Gronau, Henrik Singmann, and Eric-Jan Wagenmakers. 2020. Bridgesampling: An R package for estimating normalizing constants. Journal of Statistical Software, 92(10):1-29.

Sandra Hanne, Frank Burchert, Ria De Bleser, and Shravan Vasishth. 2015. Sentence comprehension and morphological cues in aphasia: What eyetracking reveals about integration and prediction. Journal of Neurolinguistics, 34:83-111.

Lena A Jäger, Daniela Mertzen, Julie A Van Dyke, and Shravan Vasishth. 2020. Interference patterns in subject-verb agreement and reflexives revisited: A large-sample study. Journal of Memory and Language, 111:104063.

Lena A. Jäger, Felix Engelmann, and Shravan Vasishth. 2017. Similarity-based interference in sentence comprehension: Literature review and Bayesian meta-analysis. Journal of Memory and Language, 94:316-339.

Nayoung Kwon and Patrick Sturt. 2016. Processing control information in a nominal control construction: an eye-tracking study. Journal of Psycholinguistic Research, 45(4):779-793. 
Daniel Lewandowski, Dorota Kurowicka, and Harry Joe. 2009. Generating random correlation matrices based on vines and extended onion method. Journal of Multivariate Analysis, 100(9):1989-2001.

Richard L. Lewis and Shravan Vasishth. 2005. An activation-based model of sentence processing as skilled memory retrieval. Cognitive Science, 29(3):375-419.

Paula Lissón, Dorothea Pregla, Bruno Nicenboim, Dario Paape, Mick L van het Nederend, Frank Burchert, Nicole Stadie, David Caplan, and Shravan Vasishth. 2021. A computational evaluation of two models of retrieval processes in sentence processing in aphasia. Cognitive Science, 45(4):e12956.

Andrea E Martin and Brian McElree. 2008. A contentaddressable pointer mechanism underlies comprehension of verb-phrase ellipsis. Journal of Memory and Language, 58(3):879-906.

Brian McElree. 1993. The locus of lexical preference effects in sentence comprehension: A timecourse analysis. Journal of Memory and Language, 32(4):536-571.

Brian McElree. 2000. Sentence comprehension is mediated by content-addressable memory structures. Journal of Psycholinguistic Research, 29(2):111123.

Brian McElree, Stephani Foraker, and Lisbeth Dyer. 2003. Memory structures that subserve sentence comprehension. Journal of Memory and Language, 48(1):67-91.

Paul Mätzig, Shravan Vasishth, Felix Engelmann, David Caplan, and Frank Burchert. 2018. A computational investigation of sources of variability in sentence comprehension difficulty in aphasia. Topics in Cognitive Science, 10(1):161-174.

Bruno Nicenboim and Shravan Vasishth. 2018. Models of retrieval in sentence comprehension: A computational evaluation using Bayesian hierarchical modeling. Journal of Memory and Language, 99:1-34.

Umesh Patil, Sandra Hanne, Frank Burchert, Ria De Bleser, and Shravan Vasishth. 2016. A computational evaluation of sentence processing deficits in aphasia. 40(1):5-50.

Dorothea Pregla, Paula Lissón, Shravan Vasishth, Frank Burchert, and Nicole Stadie. 2021. Variability in sentence comprehension in aphasia in German. PsyArXiv:7hfpx.

R Core Team. 2020. R: A language and environment for statistical computing. Version 4.0.2.

Daniel J Schad, Michael Betancourt, and Shravan Vasishth. 2020. Toward a principled Bayesian workflow in cognitive science. Psychological Methods, 26(1):103-126.
Nicole Stadie, Jürgen Cholewa, and Ria De Bleser. 2013. LEMO 2.0: Lexikon modellorientiert: Diagnostik für Aphasie, Dyslexie und Dysgraphie. NATVerlag, Hofheim.

Stan Development Team. 2020. RStan: the R interface to Stan. R package version 2.21.2.

Julie A Van Dyke. 2007. Interference effects from grammatically unavailable constituents during sentence processing. Journal of Experimental Psychology: Learning, Memory, and Cognition, 33(2):407.

Julie A. Van Dyke and Brian McElree. 2006. Retrieval interference in sentence comprehension. Journal of Memory and Language, 55(2):157-166.

\section{Acknowledgements}

This work was funded by the Deutsche Forschungsgemeinschaft (DFG, German Research Foundation) - Projektnummer 317633480 - SFB 1287, Project B02. PIs: Frank Burchert, Nicole Stadie, Shravan Vasishth.

\section{A Data and Code}

Data and code are available at https://bit.1y/30VVOYb.

\section{B Priors}

Equation (3) shows the priors used. These are regularizing priors (Schad et al., 2020) and allow for a broad range of parameter values. We used the same priors as Lissón et al. (2021), so that the model results could be comparable. In Lissón et al. (2021), the priors were selected by plotting the predictive prior distribution for each parameter. Plots of the prior predictive distributions can be found in the supplementary materials at https://osf.io/wkdrz.

The priors for $\alpha$ and $\gamma$ are in logit space, the rest of priors are in log space. In the modified direct-access model, $\alpha_{b}$ has the same priors as $\alpha$.

$$
\begin{aligned}
& \alpha \sim \operatorname{normal}(1,0.5) \\
& \beta_{1, \ldots, 12} \sim \operatorname{normal}(0,0.5) \\
& \mu_{0} \sim \operatorname{normal}(7.5,0.6) \\
& \gamma \sim \operatorname{normal}(-1,0.5) \\
& \delta_{0} \sim \operatorname{normal}(0,1) \\
& \sigma_{0} \sim \operatorname{normal}(0,0.5)
\end{aligned}
$$

A LKJ(2) (Lewandowski et al., 2009) prior was used for the correlation matrix of the variancecovariance matrix of the random effects. 